\title{
Metastatic Bone Marrow Tumors: Study of Nine Cases and Review of the Literature
}

\author{
Gagandeep Kaur ${ }^{1 *}$, Sabita Basu', Paramjit Kaur ${ }^{2}$ and Tanvi Sood \\ ${ }^{1}$ Department of Transfusion Medicine, Government Medical College \& Hospital, Sector 32, Chandigarh \\ ${ }^{2}$ Blood Bank, GGS Medical College, Faridkot
}

\begin{abstract}
Bone marrow examination is commonly used in the evaluation of hemato-oncological disorders and in patients with cancer of solid organs to detect metastases. This study reviews clinical, hematological and pathological data of nine cases with bone marrow metastases from solid tumors. The significance of bilateral bone marrow aspirations and biopsy has also been highlighted. Out of 784 bone marrow aspirations performed during a 69 months period, 9 patients showed metastatic bone marrow involvement. Fever (4), generalised weakness (5), loss of appetite (4) and loss of weight (4) were the main symptoms. Anemia (7) was the main hematological finding. Bilateral involvement was present in all but one patient. Fibrosis was also a significant finding on biopsy. Relevant literature review was also done. Bilateral bone marrow aspiration and trephine biopsy are an effective and cheap method for evaluating metastatic bone marrow tumors.
\end{abstract}

Keywords: Bone marrow metastases; Solid tumors

\section{Introduction}

The bone marrow is one of the organs involved by tumors that metastasize via the blood stream. In adults the tumors most often seen are carcinomas of the prostate, breast and lung, although any tumor that gives rise to blood borne metastases may infiltrate the marrow [1,2]. In children neuroblastoma, rhabdomyosarcoma, Ewing's sarcoma and retinoblastoma account for the majority of metastases [3]. This study reviews nine cases of metastatic bone marrow tumors diagnosed over a 69 months period. The aim of the study was to observe the clinical presentation and hematological profile of patients with metastatic bone marrow tumors. This study also highlights the importance of performing bilateral bone marrow aspiration and trephine biopsy in such patients and significance of correlation of the aspirate and biopsy findings.

\section{Material and Methods}

A total of 784 bone marrow aspirations performed during the period from January 1998 to September 2003 were analysed. A total of 15 bone marrow examinations were performed in patients with suspected metastatic bone marrow infiltration. The patients' clinical history, physical findings, hematological and pathological findings were reviewed retrospectively. Clinical profile included information about patient's age, sex, clinical history, physical findings and provisional diagnosis. Peripheral blood counts and blood films were reviewed. Bone marrow aspirations and bilateral trephine biopsies were performed in all the patients. The bone marrow aspiration findings were correlated with trephine biopsy results. Reticulin and Masson's trichrome (MT) stains were done on all biopsies to look for associated fibrosis.

\section{Results}

Out of 15 patients with suspected metastatic bone marrow infiltration, nine (60\%) patients showed metastatic bone marrow involvement. Out of nine patients with bone marrow metastases, one patient was of prostatic carcinoma (Figure 1), one each of gastric (Figure 2), lung and breast (post mastectomy) carcinoma (Figure 3) and in one patient carcinoma of cervix (Figure 4) was detected subsequent to the finding of bone marrow metastases. In four patients the primary site

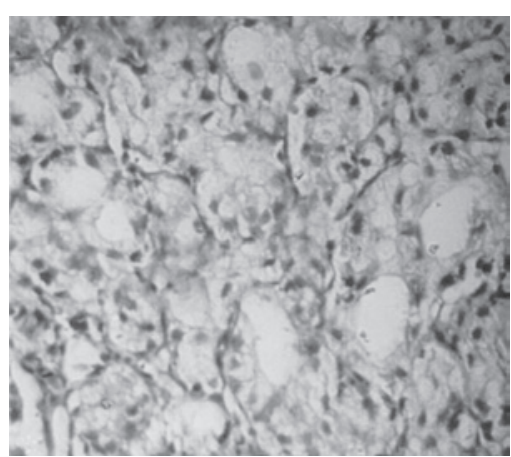

Figure 1: Glandular arrangement of metastatic cells from adenocarcinoma of prostate $(40 \mathrm{x})$.

was not known and bone marrow trephine biopsy showed metastatic tumor involvement.

History of fever was present in four out of nine patients (44.4\%), bone pains in three patients (33.3\%), loss of appetite and loss of weight in four patients each (44.4\%), low backache in two patients $(22.2 \%)$ and generalized weakness in five patients (55.5\%). One patient presented with pathological fracture of hip (11.1\%). Physical examination revealed pallor in five patients (55.5\%) lymphadenopathy in four patients and significant organomegaly in one patient (11.1\%).

Hematological profile revealed anemia in 7 patients (77.7\%), leukopenia in two and thrombocytopenia in three out of nine patients

${ }^{*}$ Corresponding author: Dr. Gagandeep Kaur, Assistant Professor, Department of Transfusion Medicine, Government Medical College \& Hospital, Sector 32 Chandigarh, India, Tel: 0172-2665253(2210); E-mail: docvpsingh@yahoo.co.in

Received August 19, 2011; Accepted October 04, 2011; Published October 24 2011

Citation: Kaur G, Basu S, Kaur P, Sood T (2011) Metastatic Bone Marrow Tumors: Study of Nine Cases and Review of the Literature. J Blood Disord Transfus 2:110. doi:10.4172/2155-9864.1000110

Copyright: (c) 2011 Kaur G, et al. This is an open-access article distributed under the terms of the Creative Commons Attribution License, which permits unrestricted use, distribution, and reproduction in any medium, provided the original author and source are credited. 


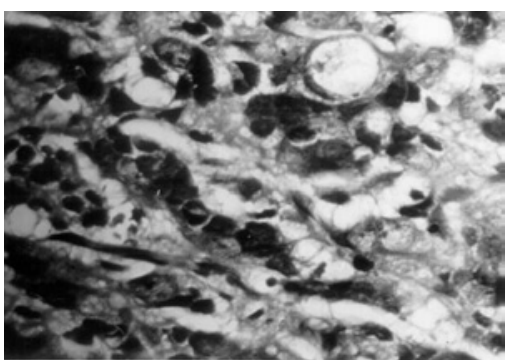

Figure 2: Bone marrow biopsy showing signet ring cells from gastric carcinoma (100x).

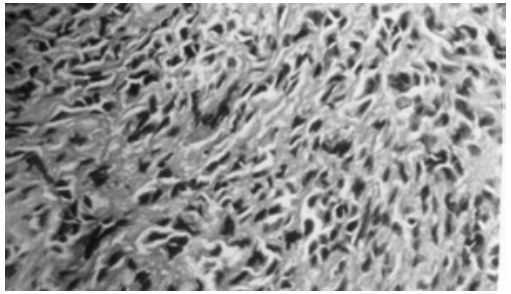

Figure 3: Infiltrating tumor cells from carcinoma of breast (20x).

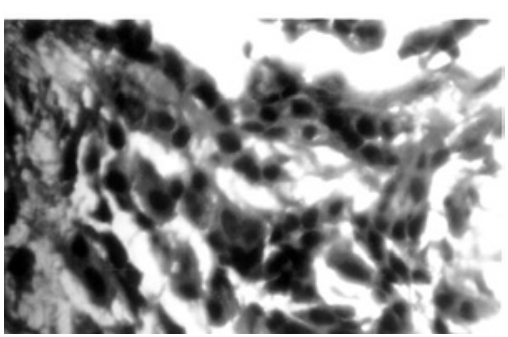

Figure 4: Metastatic cells in bone marrow biopsy from squamous cell carcinoma of cervix (100x).

(33.3\%). Three patients had bicytopenia (33.3\%) and one patient had pancytopenia (11.1\%). Examination of peripheral blood film showed leukoerythroblastic blood picture in two patients (22.2\%). However, circulating malignant cells were not found in any of the patients. Bone marrow aspirate smears were diluted in two patients and dry tap was obtained in two patients. Malignant cells were present in the aspirate smears in five patients. Bone marrow biopsy showed metastatic tumor deposits in nine patients. In eight patients (88.8\%) the involvement was bilateral and one patient had unilateral involvement. Areas of bone marrow necrosis were seen in $3(33.3 \%)$ and osteosclerosis was seen in 2 (22.2\%) patients. Reticulin stain showed fibrosis in six cases (66.6\%) in addition to metastatic tumor cells. In four patients, the primary site was not known and in one patient the primary site was detected subsequent to bone marrow examination. Bone marrow biopsy in this case showed unilateral metastasis squamous cell carcinoma which was confirmed by Papinicolau (PAP) staining. Subsequent to this gynecological examination was advised. Patient had an exophytic growth on the cervix which was diagnosed as squamous cell carcinoma on biopsy.

\section{Discussion}

Bone marrow metastases in non hematologic tumors have been reported in all types of malignancies, most common being carcinomas arising in the prostate, breast, lung and neuroblastoma. Disseminated cells from primary solid tumors are considered to be the cause of metastases formation and relapse of disease. Consequently, their detection is of high importance for staging, prognosis and decisions about adjuvant therapy. Residual disease is conventionally detected by histological evaluation of biopsy specimens [4].

Bone marrow aspirates and trephine biopsies are sensitive techniques for detecting bone marrow infiltration. Trephine biopsy is more sensitive than bone marrow aspiration and sensitivity is increased by performing bilateral biopsies or by obtaining a single large biopsy and therefore these two procedures should be regarded as complementary [5]. The detection of tumor cells in a trephine biopsy when none are demonstrable in smears of an aspirate is not uncommon. However, occasionally tumor cells are seen in aspirate smears when trephine biopsy is normal $[2,6]$.

In this study tumor cells were seen in aspirate smears in five cases and in the trephine biopsy in all nine patients. Moid et al. [7] in 2005 reported 66 cases of metastasis of solid tumors to bone marrow. They found that biopsy is positive in all cases of small carcinoma of lung and prostate carcinoma. However, in one case of infiltrating lobular carcinoma of breast, a positive bone marrow aspirate was found for which trephine biopsy was negative.

Normocytic normochromic anemia is commonly associated with infiltration of bone marrow by malignant cells. In our study anemia was detected in $77.7 \%$ and leukopenia and thrombocytopenia were detected in $33.3 \%$ cases each. The finding of anemia was similar to the study conducted by Sar R et al. [8] (66.6\%). However they detected thrombocytopenia in $83.3 \%$ and leukopenia in $50 \%$ cases with metastatic bone marrow involvement [8]. One third to half of patients with marrow infiltration may have a leukoerythroblastic blood picture. This usually correlates with the degree of bone marrow fibrosis rather than with the extent of malignant infiltration [9]. In our study, 2 out of 9 patients (22.2\%) with marrow metastasis had a leukoerythroblastic blood picture. Various studies have reported the frequency of leukoerythroblastic changes ranging from 30 to $35 \%$ $[10,11]$. Leukoerythroblastic reactions have been attributed to tumor cells "crowding out" of marrow elements due to their interaction with hematopoietic cytokines. 'Dry tap' or 'blood tap' may be obtained on repeated attempts at marrow aspiration due to reactive myelofibrosis. Dry tap was obtained in two patients. Both these patients showed reactive myelofibrosis on reticulin staining of trephine biopsies.

Marrow infiltration by metastatic tumor may be focal or diffuse. Reticulin and collagen fibrosis are commonly present and are most marked in those cases with greater degrees of marrow infiltration. Marked fibrosis is most common in carcinomas of the breast, stomach, prostate and lung $[9,12,13]$. We detected fibrosis in 6 out of 9 cases with bone marrow involvement. Frequently metastases are undifferentiated and the differential diagnosis includes poorly differentiated carcinoma, Non Hodgkins's lymphoma and malignant melanoma. In such cases immunohistochemical staining is invaluable $[14,15]$.

The two primary sites whose identification is most important because of their sensitivity to hormonal therapy are breast and prostate. In patients with relapsed or metastatic breast cancer, the finding of bone marrow metastases is reported in $3-52 \%$ of patients $[16,17,18]$. One of our patients presented with relapsed breast cancer (post mastectomy) and had extensive bone marrow infiltration. The presence of bone marrow metastases may be helpful in detecting hormonal receptors 
Citation: Kaur G, Basu S, Kaur P, Sood T (2011) Metastatic Bone Marrow Tumors: Study of Nine Cases and Review of the Literature. J Blood Disord Transfus 2:110. doi:10.4172/2155-9864.1000110

Page 3 of 3

in patients who have not had the determination done on the primary tumor [19]. Bone marrow is frequently involved by prostatic carcinoma even without evidence of skeletal disease [20]. Determination of acid phosphatase in the bone marrow is useful in detecting marrow metastases. Prostate specific antigen (PSA) is also often positive in bone marrow metastases [21]. In our study, patient with prostatic carcinoma had elevated serum acid phosphatase.

Recent interest is focussed on the biology of marrow metastases. Marrow stromal cells elaborate cytokines promoting the growth or survival of tumor cells $[22,23,24]$.

\section{Conclusion}

Bone marrow aspiration and trephine biopsy are an effective and cheap method for evaluating solid tumors metastatic to bone marrow. Such investigations are indicated when there is significant probability of bone marrow metastases and when knowledge of their presence would affect the choice of primary treatment. Moreover a clue to the primary site can also be suggested if the primary is unknown and metastatic lesions are detected in the bone marrow. Bilateral trephine biopsy is most sensitive and should be considered the gold standard when performed simultaneously with bone marrow aspiration in a patient with any malignancy potentially infiltrating the bone marrow.

\section{References}

1. Anner RM, Drewinko B (1977) Frequency and significance of bone marrow involvement by metastatic solid tumors. Cancer 39: 1337-1344.

2. Singh G, Krause JR, Breitfeld V (1977) Bone marrow examination for metastatic tumor. Cancer 40: 2317-2321.

3. Finkelstein JZ, Ekert $\mathrm{H}$, Issacs $\mathrm{H}$, Higgins $\mathrm{G}$ (1970) Bone marrow metastases in children with solid tumors. Am J Dis Child 119: 49-52.

4. Zehentner BK (2002) Detection of disseminated tumor cells: strategies and diagnostic implications. Expert Rev Mol Diagn 2: 41-48.

5. Mohanty SK, Dash S (2003) Bone marrow metastasis in solid tumors. Indian J Pathol Microbiol 46: 613-616.

6. Savage RA, Hoffman GC, Shaker K (1978) Diagnostic problems involved in detection of metastatic neoplasms by bone marrow aspirate compared with needle biopsy. Am J Clin Pathol 70: 623-627.

7. Moid F, DePalma L (2005) Comparison of relative value of bone marrow aspirates and bone marrow trephine biopsies in the diagnosis of solid tumor metastasis and Hodgkin's Iymphoma. Institutional experience and literature review. Arch Pathol Lab Med 129: 497-501

8. Sar R, Aydogdu I, Ozen S, Sevinc A, Buyukberber S (2001) Metastatic bone marrow tumours : a report of six cases and review of the literature. Haematologia 31: 215-223.

9. Rubins JR (1983) The role of myelofibrosis in malignant myelosclerosis. Cance 51: $308-311$

10. Jonsson U, Rundles RW (1951) Tumor metastases in bone marrow. Blood 6 : 16-25.

11. Chernow B, Wallner SF (1978) Variables predictive of bone marrow metastases Cancer 42: 2373-2378.

12. Kiely JM, Silverstein MN (1969) Metastatic carcinoma simulating agnogenic myeloid metaplasia. Cancer 24: 1041-1044.

13. Spector JI, Levine PH (1973) Carcinomatous bone marrow invasion simulating acute myelofibrosis. Am J Med Sci 266: 145-148.

14. Gatter KC, Abdulaziz Z, Beverly P, Corvalan JR, Ford C, et al. (1982) Use of monoclonal antibodies for the histopathological diagnosis of human malignancy. J Clin Pathol 35: 1253-1267.

15. Gatter KC, Ralfkiaer E, Skinner J, Brown D, Heryet A, et al. (1985) An immunohistochemical study of malignant melanoma and its differential diagnosis from other malignant tumors. J Clin Pathol 38: 1353-1357.

16. Ingle JN, Tormey DC, Tan HK (1978) The bone marrow examination in breast cancer: diagnostic considerations and clinical usefulness. Cancer 41: 670-674.

17. Kamby C, Guildhammer B, Vejborg I, Rossing N, Dirksen H, et al. (1987) The presence of tumor cells in bone marrow at the time of first recurrence of breast cancer. Cancer 60: 1306-1312.

18. Ceci G, Franciosi V, Passalacqua R, Diblasio B, Boni C, et al. (1988) The value of bone marrow biopsy in breast cancer at the time of first relapse : a prospective study. Cancer 61: 1041-1045

19. Papac RJ (1994) Bone marrow metastases - A review. Cancer 74: 2403-2413.

20. Rundles RW, Jonsson $U$ (1949) Metastases in bone marrow and myelophthisic anemia from carcinoma of the prostate. Am J Med Sci 218: 242-250.

21. Shah NT, Tuttle SE, Strobel SL, Gandhi L (1985) Prostatic carcinoma metastatic to bone: sensitivity and specificity of prostate specific antigen and prostatic acid phosphatase in decalcified material. J Surg Oncol 29: 265-268.

22. Sekido Y, Obata Y, Ueda R, Hida T, Suyama M, et al. (1991) Preferentia expression of c-kit proto-oncogene transcripts in small cell lung cancer. Cancer Res 51: 2416-2419.

23. Matsuda R, Takahashi T, Nakamura S, Sekido Y, Nishida K, et al. (1993) Expression of the c-kit protein in human solid tumors and in corresponding fetal and adult normal tissues. Am J Pathol 142: 339-346.

24. Roberts AB, Sporn MB (1990) The transforming growth factors betas. Handbook of experimental pharmacology. Springer-Verlag, Berlin. 